\title{
The interaction regularities of acetic and oleic acids esters and triethanolamine
}

\author{
$\underline{\text { Roman Danyliuk }}^{1}$, Anastasiia Komaretska ${ }^{1}$, Stepan Melnyk $^{1}$, Yurii Melnyk $^{1}$ \\ 1. Department of Organic Products Technology, Lviv Polytechnic National University, UKRAINE, Lviv, S. \\ Bandera str., 12, E-mail: roman.v.danyliuk@1pnu.ua
}

\begin{abstract}
The interaction regularities of ethyl and butyl esters of acetic, propionic, and oleic acids and triethanolamine have been studied. The influence of the ester type and catalyst on the process technological parameters was determined. It was found that under the same reaction conditions, oleic acid esters have higher reactivity than esters of lower aliphatic acids.
\end{abstract}

Keywords - transesterification, ammonolysis, ester, triethanolamine, catalyst.

\section{Introduction}

Ethanolamides and amino esters of fatty acids are an important class of organic compounds that function as nonionic surfactants. They have found their application as lubricants, detergents, cosmetics, paper coatings, mold removers, additives to polyethylene films, water repellents for textiles, and as additives to printing ink [1,2]. Ethanolamides and amino esters of aliphatic acids can be synthesized from ethanolamines and acyl donor molecules. Such donors are free fatty acids [3-5], fatty acid chlorides [6], vegetable oils [7] and fatty acid esters [8]. The usage of fatty acid chlorides is characterized by high process intensity. However, this method has a number of disadvantages. In particular, the synthesis is relatively expensive, and acid chlorides are corrosive and toxic substances. Their toxicity can affect the biological activity of ethanolamides in the first place. The main disadvantage of the free fatty acids usage is the formation of ionic pairs of ethanolamine and free fatty acids [9]. Due to the insoluble salt formation, the reaction becomes less efficient. Therefore, the use of fatty acid esters as acyl donors is more promising compared to other donors.

\section{Results and discussion}

This study aimed to investigate the interaction of fatty acid esters (ethyl oleate, butyl oleate, butyl propionate, and butyl acetate) with triethanolamine (grade B, ethanolamine content was $21 \mathrm{wt} . \%$ ) in the presence of catalysts cation exchange resin KU-2-8, p-toluenesulfonic acid, anion exchange resin $\mathrm{AB}-17-8$, potassium hydroxide.

The regularities of esters interaction with ethanolamines have been studied by the distillation of ethanol or butanol, formed as a result of ammonolysis and transesterification reactions. The reaction was carried out in the round bottom reactor, equipped with Dean-Stark trap and a reflux condenser. The average reaction temperature was maintained within $135 \pm 5{ }^{\circ} \mathrm{C}$. The reaction intensity was determined by the ethanol or butanol accumulation and by an amine value change of the reaction mixture. The content of alcohols was determined chromatographically. The amine value change was determined by reaction mixture titration with the sulfuric acid solution $\left(\mathrm{C}\left(1 / 2 \mathrm{H}_{2} \mathrm{SO}_{4}\right)=0,1 \mathrm{~mol} / \mathrm{dm}^{3}\right)$.

It was established that the investigated reaction mixtures have a relatively low conversion rate. In particular, under the reaction conditions of butyl propionate with ethanolamines at a molar ratio $(1,2-4,2): 1$ for a time of $140-340 \mathrm{~min}$ in the presence of $1-3,4 \mathrm{wt}$. \% of cation exchange resin KU-2-8 ester conversion is only $2,8-21,7 \%$. The feature of this reaction system (also for butyl acetate) is the presence of two liquid phases of the reagents due to ester and ethanolamines limited solubility. The presence of a heterogeneous catalyst is an additional 
reason for the low reaction rate. The accumulation of lower aliphatic alcohol in sufficient concentration homogenizes the reaction system, but afterwards the reaction slows down. It should also be noted that even though the reaction temperature is higher than the boiling point of butanol, the accumulation of this alcohol in the Dean-Stark trap is very slow. A possible reason for this is the hydroxyl groups high content in the reaction mixture and, accordingly, a significant amount of hydrogen bonds between alcohols. The type of catalyst also affects the reaction intensity. The least active catalysts are p-toluenesulfonic acid and anion exchange resin AB-178. The regularities of ethyl oleate and ethanolamines interaction were studied at a molar ratio of $1: 0,9-2$ and the content of KU-2-8 cation exchange resin in the reaction mixture of $1-3 \%$ by weight of loaded reagents. It was found that the use of a double excess of triethanolamine provides $53.6 \%$ conversion of ethyl oleate in $270 \mathrm{~min}$ of reaction. The higher degree of ester conversion can be explained by the formation of a stable emulsion between ethanolamines and oleic acid esters, which promotes better contact between the reagents.

\section{Conclusion}

It has been determined that the reaction between triethanolamine and oleic acid esters occurs more efficiently than reaction with propionic and acetic acid esters.

\section{References}

[1] Liu, K. J., Nag, A., Shaw, J. F. (2001). Lipase-catalyzed synthesis of fatty acid diethanolamides. J. Agric. Food Chem., 49, 5761-5764. doi:10.1021/jf0107858

[2] Feairheller, S., Bistline, R., Bilyk, A., Dudley, R., Kozempel, M., Haas, M. (1994). A novel technique for the preparation of secondary fatty amides. J. Am. Oil Chem. Soc., 71, 863-866. doi:10.1007/BF02540463

[3] Wang, X., Wang, X., Wang, T. (2012). Synthesis of oleoylethanolamide using lipase. J. Agric. Food Chem., 60, 451-457. doi:10.1021/jf203629w

[4] Plastina, P., Meijerink, J., Vincken, J. P., Gruppen, H., Witkamp, R., Gabriele, B. (2009). Selective synthesis of unsaturated $\mathrm{N}$-acylethanolamines by lipase-catalyzed $\mathrm{N}$-acylation of ethanolamine with unsaturated fatty acids. Lett. Org. Chem., 6, 444-447. doi : 10.2174/157017809789124885

[5] Maag, H. Fatty acid derivatives: important surfactants for household, cosmetic and industrial purposes (1984). J. Am. Oil Chem. Soc., 61, 259-267. doi:10.1007/BF02678778

[6] Kolancilar, H. (2004). Preparation of laurel oil alkanolamide from laurel oil. J. Am. Oil Chem. Soc., 81, 597-598. doi: 10.1007/s11746-006-0947-y

[7] Wang, X., Wang, T., Wang, X. (2012). An improved method for synthesis of N-stearoyl and N-palmitoylethanolamine. J. Am. Oil Chem. Soc., 89, 1305-1313. doi:10.1007/s11746-012-2017-y

[8] Khanmohammadi, M., Kojidi, M. H., Garmarudi, A. B., Ashuri, A., Soleymani, M. (2009). Quantitative monitoring of the amidation reaction between coconut oil and diethanolamine by attenuated total reflectance fourier transform infrared spectrometry. J. Surfact. Deterg., 12, 37-41. doi:10.1007/s11743-008-1101-7

[9] Tufvesson, P., Annerling, A., Hatti-Kaul, R., Adler-Creutz, D. (2007). Solvent-free enzymatic synthesis of fatty alkanolamides. Biotechnol. Bioeng., 97, 447-453. doi: $10.1002 /$ bit.21258 